TITRE: LE DÉVELOPPEMENT DE LA COMPÉTENCE À ÉCRIRE EN LANGUE PREMIÈRE ET EN LANGUE SECONDE À LA FIN DU PRIMAIRE DANS DES CONTEXTES D'INTENSIFICATION DE L'ENSEIGNEMENT DE LA LANGUE SECONDE.

Auteur(s): Olivier Dezutter, Ismail Bou Serdane, Véronique Parent (Université de SHERBROOKE), CORINNE HAIGH (UNIVERSITÉ BISHOP'S)

Publication: PeRSPECTIVES ACTUELles SUR L'APPRENTISSAGE DE LA LECTURE ET DE L'ÉCRITURE/ CONTRIBUTIONS ABOUT LEARNING TO READ AND WRITE - ACTES DU SYMPOSIUM INTERNATIONAL SUR LA LITÉRACIE À L'ÉCOLE/INTERNATIONAL SYMPOSIUM FOR EDUCATIONAL LITERACY (SILE/ISEL) 2015

PAGES: $221-241$

Directeurs: Marie-France Morin, Denis Alamargot et Carolina Gonçalves.

É́dITEUR: LES ÉDITIONS DE L'UNIVERSITÉ DE SHERBROOKE, 2016.

ISBN: 978-2-7622-0355-4

URI: HTTP://HDL.HANDLE.NET/11143/10239

DOI: HTTPS://DOI.ORG/10.17118/11143/10239 


\section{Le développement de la compétence à écrire en langue première et en langue seconde à la fin du primaire dans des contextes d'intensification de l'enseignement de la langue seconde}

Olivier Dezutter (Université de Sherbrooke), Ismail Bou Serdane (Université de Sherbrooke), Véronique Parent (Université de Sherbrooke), Corinne Haigh (Université Bishop's)

Résumé : Au Québec, un nombre de plus en plus important d'écoles organisent un apprentissage intensif de l'anglais en tant que langue seconde durant la sixième primaire. Nous présentons ici les premiers résultats d'une étude qui porte sur l'impact de cet apprentissage intensif sur le développement de la compétence d'écriture en français langue d'enseignement et en anglais langue seconde. Les élèves de cinq classes ( $n=105)$ ont passé à trois reprises (début, milieu et fin de l'année scolaire) un test standardisé (WIAT-II) visant à mesurer leur compétence en écriture dans les deux langues. Les résultats obtenus à ces tests indiquent que l'ensemble des élèves manifeste des progrès en termes de performance globale en écriture tant en français qu'en anglais. Des variations entre l'évolution des performances en L1 ou L2 sont repérables selon les sous-composantes du test. Certaines différences apparaissent également selon le modèle d'intensification de l'apprentissage auquel les élèves sont exposés.

Mots clés : anglais intensif - école primaire - compétence d'écriture - français langue d'enseignement - anglais langue seconde

Abstract: An increasing number of schools in Québec an offer students the opportunity to follow an intensive English as a second language program during their sixth year of elementary school. In this chapter we present the first results of a study that examines the impact of this intensive model of learning on the development of writing competence in French as the language of instruction and in English as a second language. Students in five classes $(n=105)$ completed a standardized test (WIAT-II) three times (beginning, middle and end of school year) to measure their writing skills in both languages. The results of these tests indicate that the students show progress in terms of overall performance in writing in both French and English. Variations in first and second language writing development are apparent when some subtest scores are examined. Certain differences are also observed when we compare the different models of intensive second language instruction that students were exposed to.

Key words: intensive English - elementary school - writing experience - French as language of instruction - English as second language 


\section{Contexte et problématique}

L'importance accordée à l'apprentissage d'une seconde, voire d'une troisième langue dans le cursus scolaire est une réalité qui a pris de l'ampleur dans les systèmes éducatifs de nombreux pays cette dernière décennie. Au Canada, le contexte politique et linguistique conduit les autorités à une centration prioritaire sur la promotion des deux langues officielles, le français et l'anglais, enseignées selon les contextes particuliers de chaque province comme L1 ou L2 (Conseil des ministres de l'éducation-Canada, 2008).

L'évolution de l'enseignement des langues secondes ou étrangères en contexte scolaire est marquée par deux tendances fortes: l'introduction de plus en plus précoce de cet enseignement (Porcher et Groux, 1998) et l'introduction de formules d'enseignement permettant un accroissement de la durée d'exposition à la $L 2$, et une intensification du contact avec celle-ci sous des formes multiples comme les programmes d'immersion, intensifs ou enrichis (Muñoz, 2012).

Au Québec, la vision de l'enseignement des langues secondes retenue pour les systèmes d'enseignement francophone et anglophone s'inscrit en partie dans cette évolution, tout en connaissant des variations importantes selon le parti politique au pouvoir. Durant les dix dernières années, le début de l'apprentissage de l'anglais en tant que langue seconde a été ramené de la quatrième à la première année du primaire (MELS, 2006) et les programmes d'enseignement intensif de l'anglais langue seconde se multiplient dans les milieux scolaires, car la demande des parents est forte pour ce type de programme (CSÉ, 2014). Celui-ci peut prendre différentes formes mais le modèle le plus répandu consiste à rassembler sur une période de 5 mois l'apprentissage de l'anglais langue seconde, et sur une période de 5 mois l'apprentissage de l'ensemble des autres matières scolaires obligatoires dont le français langue d'enseignement (MELS, 2012).

Selon la Charte de la langue française (1977), l'enseignement dans les écoles du Québec doit en effet se faire en français, ce qui rend impossible pour le secteur francophone l'implantation de la formule d'immersion qui conduirait à dispenser d'autres matières scolaires dans la langue seconde, comme cela se fait pour les programmes d'immersion en français dans les commissions scolaires anglophones ainsi que dans les autres provinces canadiennes.

Pour promouvoir ce type de programme qu'il avait un temps été envisagé de généraliser dans l'ensemble des écoles du Québec, et répondre aux inquiétudes exprimées par certains parents et groupes de pression à propos de l'impact de cette mesure sur les apprentissages des élèves en français et dans les autres matières, spécialement pour les élèves en difficulté d'apprentissage (FCPQ, 2012), le gouvernement a diffusé une synthèse de résultats de recherches soulignant le bienfondé et les bénéfices observés de l'enseignement intensif d'une langue seconde. Des études concluent en effet que les formules permettant un contact plus intensif et varié avec la L2 assurent une plus grande motivation pour cet apprentissage et de meilleurs résultats, même à plus long terme (Lightbown et Spada, 1991); que les élèves en difficulté progressent aussi dans ce type de situation pédagogique 
et en tirent des bénéfices en termes d'estime de soi et d'autonomie (Germain, Lightbown, Netten et Spada, 2004; Genesee, Geva, Dressler et Kamil, 2006); et qu'on ne dénote pas d'impact négatif sur les performances en L1 ni dans les autres matières scolaires par rapport à des élèves non exposés à une formule intensive en L2 (Lazaruk, 2007). D'autres travaux non cités par le ministère indiquent des bénéfices importants quant à l'acquisition de capacités métalinguistiques (Simard et French, 2011).

Les études consacrées à la question, majoritairement à visée évaluative, sont fondées principalement sur des données d'ordre quantitatif (résultats à des épreuves officielles le plus souvent) et examinent les impacts sur plusieurs dimensions, en fin de programme et a posteriori. Beaucoup plus rares sont les études qui s'inscrivent dans une perspective descriptive et compréhensive, centrées sur une compétence ciblée et portant sur le programme en cours d'implantation. C'est cette lacune que nous souhaitons combler en visant à apporter un éclairage inédit sur la manière dont les élèves exposés à une forme d'intensification de l'enseignement de la L2 (programme intensif ou enrichi) développent, au cours d'une année scolaire, leur compétence d'écriture en L1 et en L2.

Le choix d'une centration sur la compétence scripturale se justifie sur plusieurs plans. Pour ce qui concerne la L1, cette compétence est une des clés de la réussite scolaire (OCDE, 2000) mais les niveaux de maîtrise actuellement atteints par les élèves francophones au Québec sont source d'inquiétude (Comité d'experts, 2008). II importe dès lors de chercher à mieux comprendre comment se développe cette compétence à une étape clé de la scolarité, la dernière année de l'enseignement primaire, et dans un contexte où la pratique de l'écriture dans une L2 s'intensifie.

Pour ce qui concerne la L2, la compétence à communiquer de façon appropriée dans des contextes variés par écrit est une compétence ciblée dans les programmes d'études actuels de français et d'anglais L2 pour l'enseignement primaire (MELS, 2006 et 2009). Les attentes de fin du $3^{e}$ cycle sont similaires pour les deux langues secondes : «L'élève écrit des textes variés et bien structurés à des fins qui lui sont signifiantes. Aidé de ses pairs et de l'enseignant, il recourt le plus souvent et avec plus d'assurance à des stratégies de dépannage et d'apprentissage. II produit un texte pertinent au regard des consignes et applique les conventions linguistiques visées par le texte. À partir d'un modèle ouvert et des ressources disponibles, il livre un produit final personnalisé qui témoigne de son imagination et de sa créativité et tient compte du public cible» (MELS, 2006, p. 105). 


\section{Objectifs de la recherche}

L'objectif général de notre programme de recherche ${ }^{1}$ est de tenter de mieux comprendre de quelle manière et dans quelles conditions se développe à la fin du primaire la compétence d'écriture en langue première ( $L 1)$ et en langue seconde ( $L 2)$ dans des contextes d'intensification de l'enseignement de la langue seconde. Les objectifs spécifiques visent à :

1. Identifier les pratiques d'écriture en $L 1$ et en $L 2$ d'élèves de sixième année primaire participant à une forme d'intensification de la L2 et l'évolution de ces pratiques durant une année scolaire.

2. Déterminer le rapport à l'écriture en $L 1$ et en $L 2$ de ces élèves et l'évolution de ce rapport durant une année scolaire.

3. Déterminer le niveau de performance en écriture en $L 1$ et en $L 2$ de ces élèves et l'évolution de ce niveau de performance durant une année scolaire.

4. Explorer la relation entre les pratiques des enseignants de L1 et de L2 et l'évolution chez les élèves des différentes composantes de la compétence d'écriture examinées (pratiques, rapport et performance).

Dans le cadre de cette contribution, après avoir clarifié ce que nous entendons par « compétence scripturale » et évoqué les cadres que nous retenons à propos du développement de cette compétence en $L 1$ et en $L 2$, nous présenterons les aspects méthodo logiques ainsi que les premiers résultats relatifs à l'évolution des performances durant l'année scolaire (objectif 3). II ne s'agit encore à ce stade que de résultats partiels portant sur des données recueillies au cours de l'année scolaire 20142015 dans 5 écoles primaires du Québec. Une deuxième phase de collecte de données est en cours pour l'année scolaire 2016-2017 dans 6 autres établissements scolaires.

\section{Cadre de référence}

\section{La compétence scripturale et le rapport à l'écriture}

Selon Barbier (2003), les différentes dimensions impliquées dans l'activité d'écriture sont rangées, par la plupart des auteurs, en deux groupes distincts : les connaissances métalinguistiques, thématiques et rhétoriques d'une part, constituant l'expertise générale en production d'écrits acquise normalement dans la L1, et les connaissances linguistiques d'autre part, qui déterminent le niveau de réalisation dans la L2 ou étrangère. Multidimensionnelle, la compétence scripturale se caractérise

1. Le développement de la compétence à écrire en langue première et seconde à la fin du primaire dans des contextes d'intensification de l'enseignement de la langue seconde, recherche financée par le Conseil de recherche en sciences humaines du Canada (2014-2016), chercheur principal : Olivier Dezutter, co-chercheures : V. Parent, L. Thomas, C. Haigh, S. Lau Man Chu, C. Sabatier. 
aussi par sa variabilité et son extensivité (Dezutter, Cansigno et Silva, 2010). Des travaux récents s'inspirant de la psychologie sociale ont mis en évidence le fait que l'écriture s'accompagne d'un certain nombre de représentations, dont certaines peuvent faciliter le « passage à l'écriture » (Delamotte, Gippet, Jorro et Penloup, 2000; Delcambre et Reuter, 2002) et d'autres y faire obstacle, comme le maintien d'une représentation de l'écriture comme un don réservé à quelques privilégiés. Pour cerner la relation qu'établit un scripteur avec l'écriture, Barré de Miniac a proposé la notion de « rapport à l'écriture », définie comme « l'ensemble des relations nouées avec l'écriture, c'est-à-dire les images, représentations, conceptions, attentes et jugements qu'un sujet se forge au contact de l'écriture ellemême et d'autres utilisateurs de celle-ci » (Barré de Miniac, 1997, p. 12). Cette relation s'inscrit aussi dans une histoire plus large, construite socialement (Delcambre et Reuter, 2002). Dans le contexte qui nous intéresse, on peut dès lors penser que les différentes représentations qui circulent dans le cercle familial comme dans la société à propos de la L2 apprise et de l'utilité de sa maîtrise à l'écrit peuvent peser sur la représentation des élèves et sur leur degré d'engagement dans cet apprentissage. Nous considérons, en ce sens, que les élèves construisent un rapport à l'écriture intégrant deux dimensions (Dezutter, Cansigno et Silva, 2010) : d'une part, l'écriture en tant que mode général de communication et objet d'apprentissage scolaire, d'autre part, les particularités de l'écriture en L1 et dans la L2 qu'ils apprennent (Lyster, Collins et Ballinger (2009). À la suite de Reuter (1996), nous envisageons le développement de la compétence scripturale comme s'articulant autour de deux pôles interreliés : le pôle des représentations, investissements et valeurs d'un côté; le pôle des pratiques et performances qui associent savoirs et savoir-faire de l'autre. Les informations que nous cherchons à recueillir à propos de la compétence scripturale des élèves en L2 et en L1 dans notre programme général de recherche concernent ces différentes composantes en prenant par ailleurs en compte les particularités du passage à l'écriture de l'anglais au français et inversement. Pour cette publication, seules les données relatives aux performances sont étudiées.

\section{Les dimensions cognitive et métacognitive de l'écriture en L1 en L2}

Le modèle le plus connu relatif aux processus cognitifs impliqués dans l'acte d'écrire est celui élaboré initialement par Flower et Hayes (1981). Plus récemment, Berninger et al. (2002) ont proposé le modèle «Simple View of Writing» (SVW) qui intègre toutes les composantes du modèle précédent (planification, mise en texte, révision et publication), ainsi que la mémoire de travail, la connaissance des discours, et l'autorégulation des stratégies (Berninger et Amtmann, 2003). Les chercheurs s'entendent sur le fait que l'écriture en L2 est généralement plus coûteuse cognitivement que l'écriture en L1 (Barbier, 1998; Lefrançois, 2001; Wang et Wen, 2002; Zimmerman, 2000). La phase de planification semble souvent négligée en L2, le nombre de mots produits par unité de temps est moins élevé, les pauses sont plus fréquentes dans l'activité rédactionnelle (Barbier, 2003; De Larios, Manchon et Murphy , 2006) et la révision des productions est plus longue et fastidieuse (New, 1999). Lors de la rédaction en L2, la mémoire de travail est souvent surchargée (Barbier, 1998, 2003), car le scripteur se concentre le plus souvent sur les aspects formels de l'écrit, associés aux opérations « de bas niveau », non suffisamment automatisées, reléguant au second plan les opérations « de haut niveau » (orga- 
nisation du texte, adéquation à la situation de communication, etc.), ce qui rend généralement les textes produits en $L 2$ de moindre qualité que ceux produits en $L 1$.

Barbier (2003) a recensé plusieurs recherches qui confirment que les adultes écrivant en $\mathrm{L} 2$ s’appuient beaucoup sur leurs compétences en L1, particulièrement sur les compétences d'ordre stratégique. Cummins (1980, 2007) a émis l'hypothèse d'une compétence sous-jacente commune (common underlying proficiency) aux différentes langues apprises. Pour que des compétences de cet ordre puissent être transférées d'une langue à l'autre, il est toutefois établi (Cornaire et Raymond, 1999) qu'un seuil minimal de compétences linguistiques doit être acquis tant en L1 qu'en L2 et que les connaissances lexicales et syntaxiques jouent en ce sens un rôle clé. Cummins (1979) a montré que l'apprentissage d'une L2 a un impact positif sur les performances en L1, à condition que la compétence dans cette langue soit déjà élevée au moment de l'exposition à la L2. Ceci devrait être le cas pour la population que nous étudierons puisqu'il s'agira d'élèves qui entament leur sixième année d'apprentissage de la L1.

Dans le cas où la personne apprend plusieurs langues, il est établi que cet apprentissage ne s'effectue pas en silos distincts mais dans le cadre plutôt de multiples « circulations interlinguistiques » (Sabatier, 2008). La question du passage d'une langue à l'autre suppose une compréhension plus précise du développement langagier bi/plurilingue et du potentiel qu'il présente pour l'enseignement des langues premières et secondes (Lüdi et Py, 2003), ainsi que la prise en compte de la dimension contrastive entre les langues concernées.

\section{Méthodologie}

Visant à dresser un état de situation pour un échantillon donné sur une durée relativement longue, à savoir une année scolaire, la recherche est de type descriptif et a une dimension longitudinale. Elle a également une visée heuristique par l'exploration de nouvelles hypothèses concernant le phénomène à l'étude.

L'échantillon pour l'année 2014-2015 comprend 89 élèves scolarisés en sixième primaire dans le secteur francophone. Il s'agit d'un échantillon de convenance, constitué au départ de l'engagement volontaire des enseignants titulaires et des spécialistes de l'enseignement de la L2. L'accord des parents a été obtenu selon les règles édictées par le comité d'éthique de la recherche de l'université de Sherbrooke. Cinq classes ont participé à la recherche durant cette première année : quatre classes qui mettent en place le modèle intensif sous la forme 5 mois/5 mois (2 classes où le modèle intensif était organisé dans les 5 premiers mois de classe et 2 classes dans les 5 derniers mois) et une classe qui met en œuvre un modèle d'enseignement enrichi de l'anglais langue seconde. Dans ce cas, un temps plus important que le temps minimum requis par le programme est réservé chaque semaine pour l'apprentissage de l'anglais, et le cours de sport est donné en anglais. Afin de s'assurer de la diversité de l'échantillon, les élèves ont également été répartis en trois sous-groupes sur la base de leur 
performance en écriture en L1 à l'épreuve standardisée retenue pour l'étude (WIAT-II, Wechsler, 2005) : (1) les élèves faibles scripteurs (score d'équivalence de 95 et moins); (2) les scripteurs moyens (score d'équivalence entre 96 et 115); et (3) les scripteurs forts (score d'équivalence de 116 et plus).

Afin de déterminer le niveau de performance en écriture en $L 1$ et en $L 2$ de ces élèves et l'évolution de ce niveau de performance durant une année scolaire, nous cherchons plus spécifiquement à répondre aux questions suivantes, dans une perspective exploratoire et sur l'échantillon actuellement disponible:

1. Comment évolue la performance en L1 et en L2 au cours de l'année scolaire pour l'ensemble des élèves, de façon globale et selon les différentes dimensions évaluées (fluidité verbale à l'écrit, composition de phrases, composition de texte)?

2. Comment évolue la performance selon les deux modèles : enseignement intensif 5 mois/ 5 mois et enseignement enrichi?

3. Comment évolue la performance selon les performances initiales des élèves après la passation du premier test en L1-français (faible - moyen - fort)?

\section{Description de l'instrument de mesure et du contexte de passation}

Les performances en écriture des élèves en $L 1$ et en $L 2$ de l'ensemble de l'échantillon ont été évaluées à l'aide du sous-test Expression écrite du Test de rendement individuel de Wechsler (WIAT-II, Wechsler, 2005) à trois moments distincts au début (temps 1 : mi-septembre 2014), à la moitié (temps 2 : mi-février 2015) et à la fin de l'année scolaire (temps 3 : début juin 2015). La même épreuve est donc utilisée à ces trois moments de mesure et dans les mêmes conditions de passation afin de faciliter l'engagement des élèves dans la tâche.

Des assistants de recherche préalablement formés ont effectué la passation des tests durant le temps scolaire en accord avec les enseignants. Les tests dans les deux langues (en commençant par le test en L1- français) avaient lieu l'un après l'autre, avec un temps de pause entre les deux tests. Les consignes fournies aux élèves correspondaient aux procédures préconisées par les auteurs du test standardisé.

Le premier exercice du test mesure la fluidité verbale à l'écrit. Dans cette tâche, l'enfant doit générer, à l'écrit, le plus de mots possible correspondant à une catégorie donnée. Le second évalue l'utilisation des règles syntaxiques à l'écrit par la production de phrases. Pour ce faire, l'élève doit rédiger, à partir de plusieurs phrases ou d'images, une seule phrase bien construite qui signifie la même chose. La troisième partie du test porte sur la rédaction d'un court texte sur un sujet donné, par exemple : «mon jeu favori ». L'évaluation porte principalement sur l'organisation du texte, le vocabulaire employé, la qualité de la grammaire et le développement du thème. 
Le coefficient de fidélité pour l'ensemble du test relatif à l'expression écrite est très bon ( $r=0,82)$, les scores du WIAT-II possèdent une stabilité adéquate au fil du temps et, en termes de validité de contenu, les sous-tests évaluent de façon adéquate les concepts de rendement qu'ils sont censés évaluer (Wechsler, 2005). Cet outil présente en outre l'intérêt d'exister dans une version française et dans une version anglaise. C'est la raison pour laquelle nous avons retenu ce test, tout en prenant en compte le fait qu'il a été initialement pensé pour situer les élèves par rapport à des standards de performance en L1 et non en L2. Le WIAT-II permet de transformer les scores bruts obtenus pour tout le test (sans distinction entre les différents exercices) en scores d'équivalence (moyenne de 100 et écart-type de 15) en fonction d'un échantillon normatif évalué à trois moments pour une même année scolaire (automne, hiver et printemps).

\section{Analyse des résultats}

Pour déterminer l'évolution des performances des élèves de notre échantillon en écriture en L1 et en L2 durant une année scolaire, nous avons effectué des analyses de variance (ANOVAs) à deux facteurs intra-sujet, soit les temps de mesure (temps 1, temps 2 ou temps 3) et la langue enseignée ( L1 ou L2) sur les scores d'équivalence reflétant la performance globale en écriture et sur les scores obtenus pour chacune des sous-dimensions évaluées (fluidité verbale à l'écrit, composition de phrases, composition de texte). Les scores utilisés pour les analyses portant sur les sous-dimensions ont été transformés en pourcentages (score obtenu/score total possible X 100) afin de tenir compte des légères différences présentes entre le test en $L 1$ et en L2. Un effet d'interaction entre les variables à l'étude est ici recherché. Le cas échéant, des analyses d'effets simples en fonction de la langue enseignée ont ensuite été réalisées, avec des contrastes répétés pour la variable temps de mesure, afin de préciser les différences.

Enfin, deux ANOVAs supplémentaires ont été effectuées en ajoutant un facteur inter-sujets, et ce, afin de tenir compte soit du modèle d'enseignement (intensif ou enrichi), soit du niveau de performance initial en écriture (faible - moyen - fort). Un effet d'interaction entre l'ensemble des variables à l'étude est de nouveau recherché et le cas échéant, les effets sont décomposés par le biais d'analyses d'effets simples. 


\section{Résultats}

L'évolution des performances globales en écriture en L1 et L2 pour l'ensemble des élèves

L'ANOVA à deux facteurs intra-sujet montre a priori une différence globale en fonction des temps de mesure $\left(F(2,208)=58,58, p<0,001, \eta^{2}\right.$ partiel $\left.=0,39\right)$ et des langues enseignées $(F(1,88)=116,24$, $p<0,001, \eta^{2}$ partiel $\left.=0,57\right)$. Une tendance statistique est observée pour l'effet d'interaction $(p=0,07)$ (voir tableau 1).

Plus spécifiquement, les analyses d'effets simples montrent une évolution constante et significative des performances en $\operatorname{LI}(F(2,176)=20,10, p<0,001)$, tant entre le temps 1 et le temps 2 $(F 1,88)=12,26, p=0,001)$ qu'entre le temps 2 et le temps $3(F(1,88)=9,67, p=0,003)$. Du côté de l'écriture en $L 2$, le patron est similaire; les performances des élèves au test ont progressé d'une manière significative durant cette année scolaire $(F(2,176)=41,81, p<0,001)$. Le progrès est statistiquement significatif, tant entre le temps 1 et le temps $2(F(1,88)=33,85, p<0,001)$, qu'entre le temps 2 et le temps $3(F(1,88)=13,84, p<0,001)$. L'examen visuel des données permet en outre de supposer que la progression est plus importante entre le temps 1 et le temps 2 en L2, ce qui pourrait expliquer la tendance à l'interaction.

Enfin, les performances en $L 2$ sont significativement inférieures à celles obtenues en $L 1$ aux trois temps de mesures: temps 1 ( $t(88)=7,81, p<0,001$ ), temps $2(t(88)=7,52, p<0,001$ ) et temps $3(t(88)=7,19, p=0,001)$.

Tableau 1. Moyennes et écarts types des scores d'équivalence associés à la performance globale en écriture de l'échantillon total en L1 et en L2

\begin{tabular}{|l|l|l|l|l|l|l|l|l|l|}
\hline & \multicolumn{3}{|l|}{\begin{tabular}{l}
\multicolumn{2}{l|}{ Test WIAT de début de l'année scolaire } \\
(T1)
\end{tabular}} & \multicolumn{2}{l|}{$\begin{array}{l}\text { Test WIAT de la moitié de } \\
\text { l'année scolaire (T2) }\end{array}$} & \multicolumn{2}{l|}{$\begin{array}{l}\text { Test WIAT de la fin de } \\
\text { l'année scolaire (T3) }\end{array}$} \\
\hline & M & ET & M & ET & M & ET & \\
\hline $\begin{array}{l}\text { Performance globale } \\
\text { N=89 }\end{array}$ & L1 & 108,22 & 16,11 & 112,83 & 15,13 & 116,33 & 15,81 \\
\cline { 2 - 10 } & L2 & 95,22 & & 14,22 & 103,21 & 12,51 & 107,09 & 14,47 \\
\hline
\end{tabular}


L'évolution des performances aux sous-dimensions associées à l'écriture en L1 et L2 pour l'ensemble des élèves

Le tableau 2 présente les données descriptives (moyennes et écart types) pour les mesures de fluidité verbale à l'écrit, de composition de phrases et de composition de texte. Les résultats de l'ANOVA réalisée pour la mesure de fluidité verbale indiquent un effet principal du temps de mesure et un effet principal relatif à la langue enseignée puis une tendance statistique relative à l'effet d'interaction (voir tableau 3). Ainsi, les performances liées à la fluidité verbale à l'écrit en L1 progressent de manière significative au cours de l'année scolaire $(F(2,174)=9,49, p<00,001)$. Plus spécifiquement, les analyses indiquent une progression statistiquement significative entre le temps 1 et le temps 2 $(F(1,87)=6,00, p=0,02)$, mais pas entre le temps 2 et le temps $3(p=0,11)$. En revanche, en L2, la performance en fluidité verbale est stable au cours de l'année scolaire $(p=0,65)$. Les performances en $L 2$ sont significativement inférieures à celles obtenues en $L 1$ aux trois temps de mesures : temps 1 $(t(88)=7,13, p<0,001)$, temps $2(t(87)=9,59, p<0,001)$ et temps $3(t(88)=10,45, p<0,001)$.

Tableau 2. Moyennes et écarts des scores en pourcentage de l'échantillon total en L1 et en L2 pour la fluidité verbale à l'écrit, la composition des phrases et la composition de texte

\begin{tabular}{|c|c|c|c|c|c|c|c|}
\hline \multirow{2}{*}{\multicolumn{2}{|c|}{ M }} & \multicolumn{2}{|c|}{$\begin{array}{l}\text { Test WIAT de début de l'an- } \\
\text { née scolaire (T1) }\end{array}$} & \multicolumn{2}{|c|}{$\begin{array}{l}\text { Test WIAT de la moitié de } \\
\text { l'année scolaire (T2) }\end{array}$} & \multicolumn{2}{|c|}{$\begin{array}{l}\text { Test WIAT de la fin de } \\
\text { l'année scolaire (T3) }\end{array}$} \\
\hline & & ET & M & ET & M & ET & \\
\hline \multirow[t]{2}{*}{ Fluidité verbale (\%) } & L1 & 75,00 & 25,57 & 82,95 & 24,14 & 87,78 & 19,69 \\
\hline & L2 & 50,57 & 30,79 & 52,27 & 26,43 & 53,69 & 28,75 \\
\hline \multirow[t]{2}{*}{ Composition de phrases (\%) } & L1 & 46,82 & 17,25 & 54,02 & 17,14 & 58,84 & 19,69 \\
\hline & L2 & 32,58 & 15,32 & 41,57 & 18,87 & 49,91 & 18,74 \\
\hline \multirow[t]{2}{*}{ Composition de texte $(\%)$} & L1 & 65,99 & 11,35 & 66,44 & 11,03 & 68,89 & 9,50 \\
\hline & L2 & 57,02 & 17,66 & 67,37 & 12,25 & 71,02 & 14,58 \\
\hline
\end{tabular}


Tableau 3. Effets des analyses de variance réalisées pour les mesures de fluidité verbale à l'écrit, composition de phrases et composition de texte

\begin{tabular}{|l|l|l|l|l|l|l|}
\hline \multicolumn{2}{|l|}{ Variable } & DI & Dl erreur & F & p & $\eta 2$ partiel \\
\hline \multicolumn{2}{|l|}{ Fluidité verbale } & & & & & \\
\hline & Temps & 2 & 174 & 65,61 & 0,004 & 0,06 \\
\hline & Langue & 1 & 87 & 168,76 & $<0,001$ & 0,66 \\
\hline & Interaction & 2 & 174 & 2,71 & 0,05 & --- \\
\hline Composition de phrases & & & 176 & & & \\
\hline & Temps & 2 & 88 & 55,31 & $<0,001$ & 0,37 \\
\hline & Langue & 1 & 176 & 5,72 & $<0,001$ & 0,47 \\
\hline & Interaction & 2 & 5,39 & 0,005 & 0,06 \\
\hline Composition de texte & & 2 & 176 & 32,60 & $<0,001$ & \\
\hline & Temps & 1 & 88 & 5,16 & 0,03 & 0,27 \\
\hline & Langue & 2 & 176 & 19,09 & $<0,001$ & 0,06 \\
\hline & Interaction & & & 0,18 \\
\hline
\end{tabular}

En ce qui concerne la composition de phrases, les résultats de l'ANOVA mettent en évidence un effet principal lié au temps de mesure et à la langue enseignée, en plus d'un effet d'interaction (voir tableau 3). Plus précisément, en $L 1$, les performances augmentent dans l'ensemble de façon significative $(F(2,176)=18,39, p<0,001)$; la progression est significative entre le temps 1 et le temps 2 $(F(1,88)=17,33, p<0,001)$, puis entre le temps 2 et le temps 3 , seule une tendance statistique est observée $(p=0,07)$. En $L 2$, une progression constante des scores est relevée $(F(2,176)=46,81$, $p<0,001)$; la progression est significative entre le temps 1 et le temps $2(F(1,88)=24,34, p<0,001)$ ainsi qu'entre le temps 2 et le temps $3(F(1,88)=23,45, p<0,001)$. Encore ici, les performances en L2 demeurent significativement inférieures à celles de la $L 1$ aux trois temps de mesure : temps 1 $(t(88)=7,34, p<0,001)$, temps $2(t(88)=6,73, p<0,001)$ et temps $3(t(88)=4,01, p<0,001)$. 
Enfin, pour la rédaction de texte, l'effet d'interaction est significatif, comme les effets principaux liés au temps de mesure et à la langue enseignée (voir tableau 3). À cet égard, les résultats des tests d'effets simples indiquent, en $L 1$, une progression des performances statistiquement significative ( $F$ $(2,176)=3,70, p=0,03)$, laquelle pourrait s'observer de façon plus spécifique entre le temps 2 et le temps 3 tel qu'illustré par une tendance statistique $(p=0,06)$. Aucun changement significatif n'est observé entre le temps 1 et le temps $2(p=0,41)$. En revanche, les performances en $L 2$ augmentent tout au long de l'année $(F(2,176)=35,55, p<0,001)$; un progrès significatif est observé entre le temps 1 et le temps $2(F 1,88)=32,94 p<0,001)$ puis entre le temps 2 et le temps $3(F(1,88)=7,22, p=0,009)$. Enfin, les performances entre $L 1$ et $L 2$ diffèrent de manière significative seulement entre le temps 1 et le temps $2(t(88)=5,18, p<0,001)$, et non au temps $2(p=0,73)$ et au temps $3(p=0,12)$, ce qui indique que les performances en $L 2$ sont aussi élevées qu'en $L 1$ au milieu et à la fin de l'année scolaire.

\section{L'évolution des performances en L1 et L2 selon le modèle intensif ou enrichi}

Les résultats de l'ANOVA à deux facteurs intra-sujet et un facteur inter-sujets $(3 \times 2 \times 2)$ indiquent les effets statistiquement significatifs suivants : temps $\left(F(2,174)=15,51, p<0,001, \eta^{2} p a r t i e l=0,15\right)$; langue enseignée $\left(F(1,87)=36,09, p<0,001, \eta^{2}\right.$ partiel $\left.=0,31\right)$; et temps de mesure $X$ langue enseignée $X$ modèle d'enseignement $\left(F(2,174)=12,92, p<0,001, \eta^{2}\right.$ partiel $\left.=0,13\right)$. L'effet principal du modèle d'enseignement $(p=0,58)$; l'effet d'interaction temps de mesure X langue enseignée $(p=0,80)$; l'effet d'interaction temps de mesure X modèles d'enseignement $(p=0,14)$, et l'effet d'interaction langue enseignée $X$ modèles d'enseignement $(p=0,24)$ ne sont pas statistiquement significatifs (voir tableau 4). Ces résultats indiquent principalement un effet différentiel de l'évolution des performances en $L 1$ et en $L 2$ en fonction du modèle d'enseignement. À ce stade, ces résultats doivent toutefois être interprétés avec prudence considérant la taille restreinte de l'échantillon associé au modèle d'enseignement enrichi.

Tableau 4. Moyennes et écarts des scores globaux selon le modèle intensif ou enrichi

\begin{tabular}{|l|l|l|l|l|l|l|l|l|}
\hline \multirow{2}{*}{} & \multirow{2}{*}{} & \multicolumn{2}{|l|}{$\begin{array}{l}\text { Test WIAT de début de l'an- } \\
\text { née scolaire (T1) }\end{array}$} & \multicolumn{2}{l|}{$\begin{array}{l}\text { Test WIAT de la moitié de } \\
\text { l'année scolaire (T2) }\end{array}$} & \multicolumn{2}{l|}{$\begin{array}{l}\text { Test WIAT de la fin de l'année } \\
\text { scolaire (T3) }\end{array}$} \\
\cline { 3 - 9 } & & M & ET & M & ET & M & ET \\
\hline \multirow{2}{*}{$\begin{array}{l}\text { 5mois/5mois } \\
\text { N=78 }\end{array}$} & L1 & 109,28 & 16,23 & 112,71 & 15,01 & 116,83 & 15,79 \\
\cline { 2 - 9 } $\begin{array}{l}\text { Enrichi } \\
\text { N=11 }\end{array}$ & L2 & 94,02 & 13,64 & 103,79 & 12,32921 & 107,84 & 13,33 \\
\cline { 2 - 9 } & L1 & 100,73 & 13,59 & 113,64 & 16,68 & 112,73 & 16,21 \\
\hline
\end{tabular}




\section{L'évolution des performances en $L 1$ et $L 2$ dans le modèle 5 mois $/ 5$ mois}

Pour le modèle intensif 5 mois $/ 5$ mois, les analyses d'effets simples indiquent une progression constante en $\mathrm{L} 1$ au cours de l'année scolaire $F(2,154)=14,94, p<0,001)$, avec des progressions statistiquement significatives entre le temps 1 et le temps $2(F(1,77)=6,12, p=0,02)$, puis le temps 2 et le temps $3(F(1,77)=11,76, p=0,001)$. Le patron est similaire en $\operatorname{L2}(F(2,154)=54,63, p<0,001)$, avec des progressions statistiquement significatives entre le temps 1 et le temps $2(F(1,77)=49,35$, $p<0,001)$, puis le temps 2 et le temps $3(F(1,77)=12,55, p=0,001)$.

\section{L'évolution des performances en L1 et L2 dans le modèle enrichi}

En ce qui concerne le modèle d'enseignement enrichi, les résultats des analyses d'effets simples montrent, en $\mathrm{L} 1$, une progression significative $(F(2,20)=12,16, p<0,001)$, laquelle s'explique toutefois principalement par une progression significative des performances entre le temps 1 et le temps $2(F(1,10)=16,53, p=0,002)$. Aucune progression significative n'est observée entre le temps 2 et le temps $3(p=0,77)$. En L2, les performances sont stables et aucune progression significative n'est observée au cours de l'année ( $p=0,37)$.

\section{L'évolution des performances en L1 et L2 selon le niveau de performance initial des élèves}

Enfin, une troisième ANOVA à deux facteurs intra-sujets en fonction de la performance initiale (facteur inter-sujets) a été réalisée sur les performances globales en écriture. Dans ce contexte, l'échantillon se répartit de la façon suivante : élèves faibles scripteurs (27,0\% de l'échantillon), élèves scripteurs moyens ( $48,3 \%$ de l'échantillon) et élèves scripteurs forts (24,7\% de l'échantillon). Des résultats statistiquement significatifs sont observés pour tous les effets principaux : temps ( $F(2,172)$ $=54,786, p<0,001, \eta^{2}$ partiel $\left.=0,39\right) ;$ langue $\left(F(1,88)=138,28, p<0,001, \eta^{2}\right.$ partiel $\left.=0,62\right) ;$ perfor mance initiale des élèves $\left(F(2,86)=71,39, p<0,001, \eta^{2}\right.$ partiel $\left.=0,62\right)$. Les résultats sont également statistiquement significatifs pour les effets suivants: temps de mesure $X$ langue enseignée $(F(2,172)$ $=3,40, p=0,04, \eta^{2}$ partiel $\left.=0,04\right)$; langue enseignée $X$ performance initiale des élèves $(F(2,86)=14,08$, $p<0,001, \eta^{2}$ partiel $\left.=0,25\right)$; temps de mesure X langue enseignée $X$ performance initiale des élèves ( $F$ $(4,172)=3,31, p=0,01, \eta^{2}$ partiel $\left.=0,07\right)$. Une tendance statistique est observée pour l'effet d'interaction temps de mesure X performance initiale des élèves $(p=0,06$ ) (voir tableau 5). Globalement, ces résultats indiquent un effet différentiel de l'évolution des performances en $L 1$ et en $L 2$ en fonction de la performance initiale des élèves. 
Tableau 5. Moyennes et écarts des scores globaux en L1 et L2 selon le niveau de performance initiale en $\mathrm{L} 1$ des élèves

\begin{tabular}{|c|c|c|c|c|c|c|c|}
\hline & & \multicolumn{2}{|c|}{$\begin{array}{l}\text { Test WIAT de début de l'an- } \\
\text { née scolaire (T1) }\end{array}$} & \multicolumn{2}{|c|}{$\begin{array}{l}\text { Test WIAT de la moitié de } \\
\text { l'année scolaire (T2) }\end{array}$} & \multicolumn{2}{|c|}{$\begin{array}{l}\text { Test WIAT de la fin de l'année } \\
\text { scolaire (T3) }\end{array}$} \\
\hline & & $M$ & ET & $M$ & ET & $M$ & ET \\
\hline \multirow{2}{*}{$\begin{array}{l}\text { Faibles scripteurs } \\
\mathrm{N}=\mathbf{2 2}\end{array}$} & L1 & 89,18 & 5,69 & 100,45 & 9,57 & 102,64 & 10,22 \\
\hline & L2 & 85,13 & 9,27 & 95,95 & 7,13 & 96,86 & 10,70 \\
\hline \multirow{2}{*}{$\begin{array}{l}\text { Scripteurs moyens } \\
N=43\end{array}$} & L1 & 106,00 & 5,47 & 111,26 & 12,26 & 114,77 & 11,50 \\
\hline & L2 & 95,40 & 13,19 & 100,88 & 11,72 & 106,23 & 12,16 \\
\hline \multirow{2}{*}{$\begin{array}{l}\text { Forts scripteurs } \\
N=24\end{array}$} & L1 & 129,67 & 8,54 & 127,00 & 12,54 & 131,67 & 13,80 \\
\hline & L2 & 104,17 & 14,03 & 114,04 & 10,89 & 118,00 & 9,70 \\
\hline
\end{tabular}

L'évolution des performances en L1 et en L2 chez les élèves faibles scripteurs

Les analyses exploratoires indiquent, pour les élèves faibles scripteurs, une progression en L1 ( $F$ ( 2 , $42)=22,13, p<0,001)$, s'expliquant principalement par une progression significative entre le temps 1 et le temps $2(F(1,21)=30,96, p<0,001)$. Aucune différence statistiquement significative n'est observée entre le temps 2 et le temps 3 ( $p=0,30)$. En L2, le patron est similaire; une progression en cours d'année est observée pour les faibles scripteurs $(F(2,42)=15,47, p<0,001)$, laquelle s'explique principalement par une progression significative entre le temps 1 et le temps $2(F(1,21)=25,13, p<$ $0,001)$. Aucune différence statistiquement significative n'est observée entre le temps 2 et le temps 3 $(p=0,62)$.

\section{L'évolution des performances en L1 et en L2 chez les élèves scripteurs moyens}

La progression en L1 est constante pour les scripteurs moyens $(F(2,84)=12,15, p<0,001)$, donc significative entre le temps 1 et le temps $2(F(1,42)=8,17, p=0,007)$ puis entre le temps 2 et le temps $3(F(1,42)=4,29, p=0,05)$. Le patron est également similaire pour les scripteurs moyens en L2. L'on observe une progression constante des performances en écriture $(F(2,84)=13,80, p<0,001)$, donc significative entre le temps 1 et le temps $2(F(1,42)=6,59, p=0,001)$ puis entre le temps 2 et le temps $3(F(1,42)=11,84, p=0,001)$. 


\section{L'évolution des performances en L1 et en L2 chez les élèves scripteurs forts}

En $L 1$, aucune progression notable n'est observée pour les scripteurs forts $(p=0,20)$. Toutefois, en L2, une progression significative est notée pour les scripteurs forts $(F(2,46)=17,69, p<0,001)$, laquelle s'explique principalement par une progression significative entre le temps 1 et le temps 2 $(F(1,23)=14,07, p=0,00)$. La différence n'est pas significative entre le temps 2 et le temps $3(p=0,07)$.

\section{Conclusion}

Les analyses préliminaires et exploratoires réalisées sur un échantillon de 89 élèves permettent d'établir un certain nombre de constats en fonction des questions initialement établies :

- Il y a une progression des performances globales des élèves en écriture en français (L1) et en anglais langue seconde (L2) pour la totalité de l'échantillon tout au long de l'année scolaire. L'utilisation des scores standardisés permet ici de constater, en partie, une amélioration «normale » en cours d'année scolaire en L1, considérant que ces scores prennent en considération l'évolution normale d'un élève à trois moments au cours d'une même année scolaire. II reste que la progression observée pourrait s'expliquer par l'utilisation de manière répétée de la même mesure en l'absence d'un groupe de contrôle.

- La progression semble par ailleurs se dérouler à un rythme différent pour la L1 et pour la L2 au sens où la progression apparait plus importante en L2 entre le début et le milieu de l'année scolaire, ce qui pourrait être interprété comme une influence de l'enseignement intensif ou enrichi de la L2. En effet, au moment de la passation du test au temps 2 (troisième semaine de février), tous les élèves avaient bénéficié soit d'un 5 mois d'enseignement intensif de L2 dans la première partie de l'année, soit de trois semaines de cette forme d'enseignement, soit encore de 5 mois et demi d'enseignement enrichi.

- De façon plus spécifique, l'on note des progressions différentes en fonction des sous-dimensions de l'écriture évaluées. Une progression est observée en L1 pour la fluidité verbale et la composition de phrases surtout entre le début et le milieu de l'année scolaire. En ce qui concerne la composition de texte en LI, les progrès seraient plus saillants entre le milieu et la fin de l'année scolaire. En L2, la progression est évidente pour la composition de phrases et de texte, mais pas pour la fluidité verbale, et les performances sont similaires en L1 et en L2 au milieu et à la fin de l'année scolaire pour la composition de texte.

- En outre, les performances en L2 sont, de façon générale, inférieures aux performances en L1, ce qui peut s'expliquer, en partie du moins, par la nature du test qui est conçu pour évaluer les apprentissages en L1 mais aussi par la différence de temps d'apprentissage antérieur en L1 et en L2 en particulier pour ce qui concerne l'écriture. En outre, ces résultats pourraient aussi s'expliquer par le fait que l'écriture en L2 est généralement plus coûteuse cognitivement que l'écriture en L1 (p. ex., Barbier, 1998, Wang et Wen, 2002; Zimmerman, 2000; Lefrançois, 2001), ce qui peut se refléter du point de vue de la performance. 
- $\quad$ En ce qui concerne les modèles d'enseignement, il est possible d'observer une progression constante des performances globales en écriture, tant en L1 qu'en L2, pour les élèves s'inscrivant dans un modèle d'enseignement 5 mois/5 mois. En revanche, dans le modèle enrichi, seule une progression est observée en L1 entre le début et le milieu de l'année scolaire. Aucune progression n'est observée en L2. Ces données pourraient laisser présager un avantage du modèle 5 mois/5 mois comparativement au modèle enrichi. L'avantage pourrait résider dans le temps d'exposition à la L2 plus long dans le modèle intensif que dans le modèle enrichi. Se référant aux travaux de Curtain (2000), Johnstone (2002) souligne en effet l'incidence du temps réellement investi (temps passé à apprendre et à utiliser la langue cible) sur l'efficacité de l'apprentissage d'une langue seconde ou étrangère. Toutefois, il faut également prendre en considération la différence de la taille de l'échantillon associée aux deux modèles (plus petite pour le modèle enrichi), ce qui pourrait avoir une incidence sur les résultats. De plus, de manière descriptive, les élèves s'inscrivant dans le modèle enrichi présentent, de façon initiale, des performances plus élevées en L2 comparativement aux élèves du modèle 5 mois/5mois, ce qui pourrait s'expliquer par les critères de sélection des élèves pour faire partie de ce modèle. En outre, la présence de très bonnes capacités initiales en L2 pourrait occasionner un effet plafond, ce qui laisse ensuite moins de chance de détecter une progression marquée.

- $\quad$ Sur la base des données disponibles, le fait de présenter une performance initiale en écriture en L1 faible, moyenne ou forte, influence la progression des performances en L1 et en $L 2$. Les résultats indiquent que les performances estimées selon leur score global progressent dans tous les cas en L2, peu importe la performance initiale, mais principalement entre le début et le milieu de l'année scolaire pour les scripteurs faibles et les scripteurs forts. En L1, les performances des élèves forts scripteurs restent stables au cours de l'année scolaire, ce qui peut s'expliquer, du moins en partie, justement par la présence de très bonnes capacités initiales, laissant par le fait même moins de place à l'amélioration dans le cadre du test proposé (effet plafond). Les élèves faibles scripteurs progressent pour leur part en L1 de manière plus importante entre le début et le milieu de l'année.

À partir de ces constats, nous pouvons à ce stade conclure que l'enseignement intensif de L2 n'a pas d'incidence négative sur les performances en écriture des élèves en L1 et en L2 en général, ce qui permet, en partie, d'appuyer les résultats d'études antérieures présentées dans notre cadre de référence. II demeure qu'à ce stade de notre recherche, ces résultats doivent être interprétés avec nuance étant donné le caractère restreint de ce premier échantillon, limitant la puissance des analyses statistiquement. Des analyses subséquentes sur la base d'un plus grand échantillon permettront de mieux comprendre l'influence de certaines variables, dont particulièrement le modèle d'enseignement et le niveau de performance initial en écriture.

Les autres volets de cette recherche en cours, à savoir l'analyse de mini-entrevues avec des élèves des différents groupes et l'analyse linguistique approfondie de leurs productions aux tests, apporteront par ailleurs des éclairages complémentaires quant à la manière dont les différentes dimensions du rapport à l'écriture dans les deux langues évoluent dans ce type de contexte d'apprentissage. 


\section{Bibliographie}

Barbier, M.-L. (1998). Rédaction en langue première et en langue seconde : comparaison de la gestion des processus et des ressources cognitives. Psychologie française, 43(4), 361-370.

Barbier, M. L. (2003). Écrire en L2 : bilan et perspectives des recherches.Arob@se, 1(2),6-21. Récupéré du site de l'auteure: https://www.univ-rouen.fr/arobase/v7/barbier.pdf

Barré de Miniac, C. (1997). La famille, l'école et l'écriture. Paris, France: INRP.

Berninger, V. et Amtmann, D. (2003). Preventing written expression disabilities through early and continuing assessment and intervention for handwriting and/or spelling problems: Research into practice. Dans H. Swanson, K. Harris, and S. Graham (dir.), Handbook of learning disabilities (p. 323- 344). New York, NY: The Guilford Press.

Berninger, V. W., Vaughan, K., Abbott, R. D., Begay, K., Coleman, K. B., Curtin, G., ... Graham, S. (2002). Teaching spelling and composition alone and together: Implications for the simple view of writing. Journal of Educational Psychology, 94(2), 291-304.

Conseil des ministres de l'éducation (Canada) (CMEC). (2008). Rapport intérimaire pancanadien sur les langues officielles dans l'enseignement, de 2005-2006 à 2006-2007. Toronto, Canada: Gouvernement du Canada.

Conseil supérieur de l'éducation (CSÉ). (2014). L'amélioration de l'enseignement de l'anglais, langue seconde, au primaire : un équilibre à trouver. Québec, Canada.

Cornaire, C. et Raymond, P.-M. (1999). La production écrite. Paris, France: CLE International.

Cummins, J. (1979). Cognitive/academic language proficiency, linguistic interdependance, the optimum age question and some other matters. Working papers on bilingualism, 19, 197-202.

Cummins, J. (1980). The Cross-Lingual Dimensions of Language Proficiency: Implications for Bilingual Education and the Optimal Age Issue, TESOL Quarterly, 14(2), 175-187.

Cummins, J. (2007). Rethinking monolingual instructional strategies in multilingual classrooms. Canadian Journal of Applied Linguistics 10(2), 221-240.

Curtain, H. (2000). Time as a factor in early start programmes. Dans J. Moon \& M. Nikolov. (dir.). Research into teaching English to young learners. (Pécs: University of Pécs Press). 87-120.

De Larios, J.-R., Manchon, R.-M. et Murphy, L. (2006). Generating text in native and foreign language writing: A temporal analysis of problem-solving formulation processes. Modern Language Journal, 90(1), 100-114.

Delamotte, R., Gippet, F., Jorro, A. et Penloup, M-C. (2000). Passages de l'écriture: un défi pour les apprenants et les formateurs. Paris, France: Presses universitaires de France.

Delcambre, I. et Reuter, Y. (2002). Texte de présentation. Pratiques, 2(6), 113-114. 
Dezutter, O., Cansigno, Y. et Silva, H. (2010). Un cadre théorique pour penser le développement de la compétence scripturale en langue seconde ou étrangère. Dans Y. Cansigno, O. Dezutter, H. Silva et F. Bleys (dir.), Défis d'écriture. Développer la compétence scripturale en français langue seconde ou étrangère à l'université. Mexico : Presses de l'UAM-Azcapotzalco, 21-33.

Fédération des comités de parents du Québec (FCPQ). (2012). L'enseignement intensif de l'anglais, L2. Résultats du sondage mené auprès des membres de la FCPQ et de la consultation faite auprès des parents du FPEHDAA.

Genesee, F., Geva, E., Dressler, C. et Kamil, M. (2006). Synthesis: Cross-linguistic relationships. Dans D. August et T. Shanahan (dir.). Developing Literacy in Second-Language Learners. Report of the National Literacy Panel on Language-Minority Children and Youth. Mahwah, NJ: Lawrence Erlbaum.

Germain, C., Lightbown, P.M., Netten, J. et Spada, N. (2004). Intensive French and intensive English: Similarities and differences. Canadian Modern Language Review, 60(3), 409-430.

Hayes, J.R. et Flower, L.S. (1980). « Identifying the organization of writing processes », in Gregg, L.W. \& Steinberg, E.R. (dir.), Cognitive processes in writing. Hillsdale, NJ,Lawrence Erlbaum, 3-30. Johnstone, R. (2002). À propos du «facteur de l'âge » : quelques implications pour les politiques linguistiques, Conseil de l'Europe.

Lazaruk, W. (2007). Linguistic, academic, and cognitive benefits of French immersion. Revue canadienne des langues vivantes, 63(5), 605-628. Lefrançois, G. R. (2001). Of Children: An Introduction to Child and Adolescent Development. Belmont, CA: Wadsworth.

Lightbown, P. M. et Spada, N. (1991). Long-term study of intensive ESL teaching in primary school. Canadian Modern Language Review, 48, 1-17.

Lüdi, G. et Py, B. (2003). Etre bilingue. Berne, Suisse: Peter Lang.

Lyster, R., Collins, L. et Ballinger, S. (2009). Linking languages through a bilingual read-aloud project. Language Awareness, 18, 366-383.

Mieux soutenir le développement de la compétence à écrire (2008). Rapport du Comité d'experts sur l'apprentissage de l'écriture. Gouvernement du Québec.

Ministère de l'Éducation, du Loisir et du Sport (MELS). (2006). Programme de formation de l'école québécoise. Québec, Canada : Gouvernement du Québec.

Ministère de l'Éducation, du Loisir et du Sport (MELS). (2009). Programme de formation de l'école québécoise: Progression des apprentissages au primaire. Québec, Canada : Gouvernement du Québec.

Ministère de l'Éducation, du Loisir et du Sport (MELS). (2012). L'organisation scolaire dans un contexte d'enseignement intensif de l'anglais L2. Québec, Canada: Gouvernement du Québec.

Muñoz, C. (éd.) (2012). Intensive Exposure Experiences in Second Language Learning. Bristol: Multilingual Matters. 
New, E. (1999). Computer-aided writing in French as a foreign language: A qualitative and quantitative look at the process of revision. Modern Language Journal, 83(1), 80-97.

Organisation de Coopération et de Développement Économiques (OCDE). (2000). La littératie à l'ère de l'information. Paris, France : OCDE.

Porcher, L. et Groux, D. (1998). L'apprentissage précoce des langues. Paris, France: Presses universitaires de France.

Reuter, Y. (1996). Enseigner et apprendre à écrire. Paris, France: ESF.

Sabatier, C. (2008). Ethnic and national identity among second-generation immigrant adolescents in France: the role of social context and family. Journal of Adolescence, 31(2), 185-205.

Simard, D. et French, L. (2011). Lien entre l'objet des verbalisations langagières d'élèves de sixième année du primaire et leurs productions écrites en anglais langue seconde. Revue canadienne de linguistique appliquée, 14, 141-169.

Wang, W. et Wen, Q. (2002). L1 use in the L2 composing process: An exploratory study of 16 Chinese EFL writers. Journal of second language writing, 11(3), 225-246.

Wechsler, D. (2005). WIAT-II: Test de rendement individuel de Wechsler - Deuxième édition. Toronto, Canada: Pearson Canada Assessment.

Zimmerman, R. (2000). L2 writing: subprocesses, a model of formulating and empirical findings. Learning and Instruction, 10(1), 73-99. 